\title{
PREVENTION OF LOW CARDIAC OUTPUT SYNDROME (LCOS) IN NEONATES UNDERGOING OPEN HEART SURGERY: A PILOT-PHASE II STUDY ABOUT THE EQUIVALENCE OF TWO INODILATORS (INDS)
}

\author{
A. Pellicer ${ }^{1}$, J. Riera ${ }^{1,2}$, P. Lopez-Ortego ${ }^{1}$, M.C. Bravo $^{1}$, R. Madero $^{3}$, J. Perez-Rodriguez ${ }^{1}$, C. Labrandero ${ }^{4}$, J.

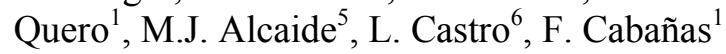 \\ ${ }^{1}$ Dept. of Neonatology, La Paz University Hospital, Autonoma University, ${ }^{2}$ Bioinstrumentation and \\ Nanomedicine Lab, Escuela Tecnica Superior de Ingenieria de Telecomunicaciones, Universidad \\ Politecnica, ${ }^{3}$ Biostatistics Unit, ${ }^{4}$ Dept. of Paediatric Cardiology, ${ }^{5}$ Central Haematology and Biochemistry \\ Lab, ${ }^{6}$ Dept. of Paediatric Anesthesiology, La Paz University Hospital, Autonoma University, Madrid, Spain
}

Background: Neonates are at particular risk of suffering surgery-related LCOS, characterized by impaired myocardial contractility and the peripheral effects of ischemia/reperfusion on endothelium. INDs are strongly recommended although based on suboptimal studies.

Aims: Systematic approach to dose-dependent haemodynamic effects of continuous i.v. infusion of Milrinone (MR) and Levosimendan (LEVO), starting before cardiopulmonary bypass .

Methods: Intervention (first 48h, blinded): step- increase in INDs dose $\left(D_{1}\right.$ : intraoperatively; $D_{2}$ : on NICU admission; $D_{3}: 2 \mathrm{~h}-48 \mathrm{~h}$ from admission). INDs withdrawal: LEVO at $48 \mathrm{~h}$; MR beyond $48 \mathrm{~h}$ as per attending physician criteria. Continuous, time-locked physiological and near-infrared spectroscopy (cerebralNIRSc/thigh-NIRSp) data recording during the first $24 \mathrm{~h}$ (T-1), at 48h (T-2) and 96h (T-3) post-surgery. Blood samples for biochemistry and pharmacokinetics (PKs). Serial echocardiography and cranial-Doppler ultrasound studies.

Results: 20 infants [postnatal age: MR, 13 (10) days; LEVO, 15 (9) days] were randomized [(MR=9; $D_{1} 0.5$ $\left.\left.D_{2} 0.75-D_{3} 1 \mathrm{mg} / \mathrm{k} / \mathrm{min}\right) ;\left(\mathrm{LEVO}=11 ; D_{1} 0.1-D_{2} 0.15-D_{3} 0.2 \mathrm{mg} / \mathrm{k} / \mathrm{min}\right)\right]$. MR showed lower $\mathrm{pH}$ and higher glycemia and more need for other inotropes during the first hours post-surgery. MR and LEVO showed no differences in time-related changes on NIRSc (increased tissue oxygenation) or blood pressure (decreased diastolic pressure) in T-1. However, groups differed in NIRSc-derived variables from T-1 to T-3. INDs withdrawal at T-3 was $37 \%$ in MR vs $91 \%$ in LEVO. PKs (LEVO and metabolites) were successfully analyzed.

Conclusions: LEVO is well tolerated in critically ill neonates. Potential advantages of LEVO related to dose regimen. 